\title{
Perfil Epidemiológico dos Pacientes com Câncer de Cabeça e Pescoço em um Centro Oncológico no Sul do Brasil
}

doi: https://doi.org/10.32635/2176-9745.RBC.2020v66n1.455

\author{
Epidemiological Profile of Patients with Head and Neck Cancer at a Cancer Center in Southern Brazil \\ Perfil Epidemiológico de Pacientes con Cáncer de Cabeza y Cuello en un Centro de Oncología en el Sur de Brasil
}

Fernanda Alessandra da Silva'; Suellen Cristina Roussenq²; Michelle Gonçalves de Souza Tavares ${ }^{3}$; Cristiana Pezzi Franco de Souza ${ }^{4}$; Carolina Barreto Mozzini5; Magnus Benetti ${ }^{6}$; Mirella Dias ${ }^{7}$

Resumo

Introduçáo: $\mathrm{O}$ câncer de cabeça e pescoço aumentou significativamente na última década. Objetivo: Determinar o perfil epidemiológico dos pacientes com câncer de cabeça e pescoço atendidos em um Centro Oncológico de referência no Sul do Brasil no período de janeiro de 2013 a dezembro de 2018. Método: Estudo de caráter descritivo e retrospectivo realizado no Centro de Pesquisas Oncológicas (Cepon). Resultados: Foram obtidos dados preliminares de 133 pacientes. O gênero masculino foi o mais prevalente $(65,4 \%)$, com média de idade acima dos 50 anos, baixa escolaridade (40\%), baixa renda (77,3\%), sendo sua grande maioria tabagistas (72\%) e etilistas (58,1\%). A regiāo de cavidade oral $(26,3 \%)$ foi o sítio mais prevalente, a cirurgia mais realizada foi a tireoidectomia total (19,4\%) e o estadiamento mais observado foi T2 $(30,8 \%)$ N2 (41,1\%). Foram encontradas complicaçôes pós-radioterapia como a radiodermite (82,7\%) e, pósquimioterapia, náuseas $(81 \%)$. As fibroses cicatriciais foram mais frequentes após a cirurgia $(18,2 \%)$, sendo avaliadas no pós-operatório tardio. Conclusáo: Caracterizar o perfil dos pacientes com câncer de cabeça e pescoço permite conhecer os diversos acometimentos advindos do tratamento e possibilita, dessa maneira, maior qualidade e direcionamento das açôes de reabilitaçáo.

Palavras-chave: Neoplasias de Cabeça e Pescoço; Perfil de Saúde; Oncologia.

\section{Abstract}

Introduction: Head and neck cancer has increased significantly in the last decade. Objective: To determine the epidemiological profile in patients with head and neck cancer treated at an oncological referral center in Brazil Southern region from january 2013 to december 2018. Method: Descriptive and retrospective study carried out at CEPON (Oncology Research Center). Results: Preliminary data were obtained from 133 patients. Male gender was the most prevalent $(65.4 \%)$, with mean age above 50 years, low education $(40 \%)$, low income $(77.3 \%)$, many of them were smokers $(72 \%)$ and alcoholics (58.1\%). The oral cavity region $(26.3 \%)$ was the most prevalent site, the most performed surgery was total thyroidectomy (19.4\%) and the most observed staging was T2 (30.8\%) and N2 (41.1\%). Post-radiotherapy and post-chemotherapy complications as radiodermatitis $(82.7 \%)$ and nausea $(81 \%)$, respectively, were found. Scarring fibrosis was more frequent after surgery (18.2\%) and were evaluated in the late postoperative period. Conclusion: Characterizing the profile of patients with head and neck cancer allows to know the several affections resulting from the treatment to ensure better quality and focus of rehabilitation actions.

Key words: Head and Neck Neoplasms; Health Profile; Medical Oncology.

\section{Resumen}

Introducción: El cáncer de cabeza y cuello ha aumentado significativamente en la última década. Objetivo: Determinar el perfil epidemiológico en pacientes con cáncer de cabeza y cuello tratados en un centro de referencia en el sur de Brasil desde enero de 2013 hasta diciembre de 2018. Método: Estudio descriptivo y retrospectivo realizado en el Centro de Investigación Oncológica (Cepon). Resultados: Se obtuvieron datos preliminares de 133 pacientes. El sexo masculino fue el más prevalente (65.4\%), con una edad promedio de más de 50 años, baja escolaridad (40\%), bajos ingresos (77.3\%), la mayoría de ellos fumadores (72\%) y alcohólicos. (58.1\%). La región de la cavidad oral (26.3\%) fue el sitio más prevalente, la cirugía más realizada fue la tiroidectomía total $(19.4 \%)$ y la estadificación más observada fue T2 (30.8\%) y N2 (41.1\%). Se encontraron complicaciones posteriores a la radioterapia, como radiodermatitis $(82,7 \%)$ y después de la quimioterapia, náuseas $(81 \%)$. La fibrosis cicatricial fue más frecuente después de la cirugía $(18,2 \%)$ y se evaluó en el postoperatorio tardío. Conclusión: La caracterización del perfil de los pacientes con cáncer de cabeza y cuello nos permite conocer las diversas afecciones que surgen del tratamiento y, por lo tanto, permitir una mayor calidad y dirección de las acciones de rehabilitación.

Palabras clave: Neoplasias de Cabeza y Cuello; Perfil de Salud. Oncología Médica.

\footnotetext{
1 Universidade do Sul de Santa Catarina (Unisul). Florianópolis (SC), Brasil. Orcid iD: https://orcid.org/0000-0002-9975-6793

${ }^{2}$ Centro de Pesquisas Oncológicas (Cepon). Hospital Florianópolis. Universidade do Estado de Santa Catarina (Udesc). Faculdade Inspirar. Florianópolis (SC), Brasil. Orcid iD: https://orcid.org/0000-0001-8202-6244

3 Unisul. Udesc. Universidade Federal de Santa Catarina (UFSC). Florianópolis (SC), Brasil. Orcid iD: https://orcid.org/0000-0002-5390-8739

${ }^{4}$ Cepon. Florianópolis (SC), Brasil. Orcid iD: https://orcid.org/0000-0002-6862-6275

${ }^{5}$ AC Camargo Cancer Center. Universidade de São Paulo. São Paulo (SP), Brasil. Hospital de Clínicas de Passo Fundo. Passo Fundo (RS), Brasil. Orcid iD: https://orcid. org/0000-0003-4360-2936

${ }^{6}$ Udesc. Florianópolis (SC), Brasil. University of North Carolina at Chapel Hill. UNC, EUA. Universidade Federal do Rio Grande do Sul (UFRGS). Rio Grande do Sul (RS), Brasil. Orcid iD: https://orcid.org/0000-0002-0079-255X

${ }^{7}$ Cepon. Unisul. Udesc. UFSC. Florianópolis (SC), Brasil. Orcid iD: https://orcid.org/0000-0002-2019-3563

Endereço para correspondência: Suellen Cristina Roussenq. Rua Odillon Gallotti, 433-Capoeiras. Florianópolis (SC), Brasil. CEP 88070-480. E-mail: suellen.roussenq@cepon.org.br
} 


\section{INTRODUÇÃO}

O câncer de cabeça e pescoço é um termo coletivo usado para definir as neoplasias que acometem o trato aerodigestivo superior, no qual se incluem as regióes de cavidade oral, faringe, laringe e tireoide. Aproximadamente $40 \%$ dos cânceres de cabeça e pescoço ocorrem na região de cavidade oral (assoalho bucal, língua, base da língua, palato duro e lábios); $15 \%$ na faringe (orofaringe, hipofaringe e nasofaringe); $25 \%$ na laringe; e o restante em glândulas salivares e tireoide ${ }^{1,2}$.

A incidência de câncer na regiáo de cavidade oral aumentou durante a última década, principalmente na base da língua e amígdalas, essencialmente em pessoas com idade inferior a 45 anos, em razão da alta prevalência do papilomavírus humano (HPV), que vem sendo considerado um fator de risco cada vez mais importante para esses tipos de cânceres. Tal aumento se justifica também pela prática frequente de sexo oral e do número elevado de parceiros sexuais ${ }^{3,4}$.

No câncer de cabeça e pescoço, os fatores de risco genéticos e ambientais contribuem para sua etiologia, como o consumo de álcool e tabaco, que são os fatores de risco mais relacionados ao acometimento do trato aerodigestivo superior ${ }^{4,5}$. No caso do câncer de tireoide, diversas são as causas ditas como fatores de risco, sendo elas a exposição à radiação, condiçôes hormonais, obesidade, história familiar de câncer e ingestão de alimentos iodados ${ }^{6}$.

O tratamento desses tipos de câncer pode incluir a ressecção cirúrgica, quimioterapia, radioterapia ${ }^{7}$, hormonioterapia ou a combinação de mais de uma forma de tratamento ${ }^{8}$, acarretando alteraçóes na funcionalidade e na qualidade de vida9. Por esse motivo, a atenção ao paciente portador de câncer de cabeça e pescoço deve ser uma abordagem multidisciplinar, com equipe composta por médicos, cirurgióes-dentistas, fisioterapeutas, enfermeiros, fonoaudiólogos, entre outros profissionais ${ }^{10}$.

As modalidades de tratamento para esses pacientes podem trazer prejuízos à qualidade de vida ${ }^{8}$, gerando alteraçóes como mucosites (inflamaçóes da mucosa oral), xerostomia (boca seca), fadiga e radiodermites (alteraçóes da pele), que acarretam prejuízos à mastigação, deglutiçấo e até mesmo a fala?.

Essas alteraçốes ocasionam, na maioria das vezes, perda de peso e desnutriçáo por causa das morbidades geradas pelo tratamento, provocando um enorme declínio funcional ${ }^{9}$. Entre as alteraçóes e declínios funcionais causados pelos tratamentos do câncer de cabeça e pescoço, há ainda o linfedema, a síndrome do ombro caído, paralisias faciais, trismo (redução da abertura bucal) e paralisia das cordas vocais ${ }^{7,11}$.
O câncer de cabeça e pescoço é induzido por uma combinação de fatores, inclusive pela Regiāo ${ }^{12}$; ou seja, existe uma discrepância observada nos dados epidemiológicos publicados da doença nas diversas Regiōes do Brasil, podendo ser pela carência dos registros de dados ${ }^{12}$, demonstrando que existem poucos dados da doença na literatura e que tais valores são subestimados ${ }^{13}$. Estudos epidemiológicos são fundamentais para as informaçóes em saúde e para profissionais da área da saúde, permitindo o início das açôes de saúde pública, garantindo planejamentos e medidas específicas de prevenção, principalmente para a população estudada, cujo atraso do diagnóstico é a principal causa de um prognóstico ruim ${ }^{13-15}$.

Torna-se, desse modo, prioridade, a pesquisa de informaçóes sobre o perfil dos pacientes com câncer de cabeça e pescoço para uma melhor assistência, elaboração de estratégias de prevenção, um maior conhecimento sobre as principais morbidades, auxílio no diagnóstico precoce e reabilitação ainda em estágios iniciais, melhorando, assim, o prognóstico dessa população. Dessa forma, o presente estudo objetivou determinar o perfil dos pacientes com câncer de cabeça e pescoço atendidos em um Centro Oncológico de referência no Sul do Brasil.

\section{MÉTODO}

Trata-se de um estudo de caráter descritivo e retrospectivo realizado no Centro de Pesquisas Oncológicas (Cepon) na cidade de Florianópolis, SC, Brasil, e que teve como populaçáo-alvo pacientes oncológicos de cabeça e pescoço, atendidos na referida instituição entre janeiro de 2013 a dezembro de 2018.

Realizou-se um Censo com todos os prontuários arquivados dos pacientes com diagnóstico de neoplasias de cabeça e pescoço, atendidos no Serviço de Fisioterapia, com idade acima de 18 anos no período estudado. Não foram excluídos os prontuários que possuíssem dados incompletos.

Para a coleta dos dados, foi utilizado o prontuário eletrônico do Cepon de SC. A instituição utiliza um sistema de software de gestáo hospitalar no qual são armazenadas todas as informaçóes referentes ao paciente como dados sociodemográficos, bem como dados relacionados à doença, tratamento clínico, cirúrgico e fisioterapêutico.

Utilizou-se uma ficha de avaliaçáo, criada pelos pesquisadores, para transcrever os dados coletados dos prontuários eletrônicos, são eles: variáveis sociodemográficas (idade, cidade, sexo, etnia, escolaridade, ocupaçáo, estado civil, renda familiar e classe social), estilo de vida, história clínica/diagnóstico, antecedentes clínicos, tipo de câncer 
de cabeça e pescoço, comorbidades, tratamento realizado (cirurgia, quimioterapia, radioterapia) e complicaçóes geradas pelo tratamento.

Os tumores foram classificados de acordo com o sistema de estadiamento preconizado pela União Internacional Contra o Câncer (UICC), denominado Sistema TNM de Classificação dos Tumores Malignos ${ }^{12}$.

Os dados do presente estudo foram coletados somente após aprovaçáo do Comitê de Ética em Pesquisa sob o número de parecer 3.215.586. Realizou-se a coleta nas dependências do Cepon de SC no período de janeiro a abril de 2019.

Os dados foram analisados no programa Statistical Package for the Social Sciences (SPSS ${ }^{\circ}$ ) versão 20.0. A análise descritiva das variáveis contínuas foi realizada por meio das médias, medianas e desvio-padrão. As variáveis categóricas foram analisadas por meio das frequências absoluta e relativa.

\section{RESULTADOS}

Foram obtidos dados preliminares de 133 prontuários de pacientes atendidos entre os anos de 2013 a 2018. Quanto ao gênero, 65,4\% ( $\mathrm{n}=87)$ eram do sexo masculino, com média de idade foi $57,9 \pm 12,5$ anos, majoritariamente brancos $(94, n=125)$. Do total, a maioria, 40\% $(n=50)$, possuía ensino fundamental incompleto e realizava trabalhos manuais e braçais, possuindo renda familiar caracterizada como baixa (77,3\%, 58 indivíduos). Grande parte dos indivíduos era tabagistas $(72 \%, \mathrm{n}=85)$ e etilistas $(58,1 \%, \mathrm{n}=61)$, com tempo médio de exposição de 34 e 33 anos, respectivamente. Muitos pacientes apresentavam hipertensão arterial sistêmica, diabetes mellitus ou ambas as comorbidades. Demais características sociodemográficas podem ser observadas na Tabela 1.

Os dados apresentados na Tabela 2 descrevem as características clínicas e cirúrgicas da amostra, contendo o estadiamento dos tumores.

Quanto ao tipo de tumor primário, observou-se maior representatividade na cavidade oral $(26,3 \%, n=35)$, tireoide $(25,5 \%, \mathrm{n}=34)$ e orofaringe $(24,0 \%, \mathrm{n}=32)$, com local de maior prevalência de metástase a região cervical $(42,3 \%, \mathrm{n}=11)$ (Tabela 3).

A radioterapia foi o método mais empregado em 76,7\% ( $\mathrm{n}=102)$ da amostra, em sua maioria, associada ao tratamento cirúrgico $(83,5 \%, n=111)$. O tratamento cirúrgico predominante foi a tireoidectomia total $(19,4 \%, n=21)$ e alguns pacientes necessitaram de complementação com a radioiodoterapia (12\%, $\mathrm{n}=16$ ). Apenas 18 pacientes precisaram de uma nova cirurgia durante o tratamento, sendo a mais comum a glossectomia $(16,7 \%, \mathrm{n}=3)$ (Tabela 4).
Tabela 1. Características sociodemográficas e clínicas dos pacientes com câncer de cabeça e pescoço ( $n=133$ )

\begin{tabular}{lccc} 
& Variável & N & $\%$ \\
\hline Idade* & $57,98 \pm 12,54$ \\
\hline Sexo & & \\
Masculino & 87 & 65,4 \\
Feminino & 46 & 34,6 \\
Total & 133 & 100,0 \\
\hline
\end{tabular}

\section{Cor da pele}

Branca

12594,0

$\begin{array}{lll}\text { Não branca } & 8 & 6,0\end{array}$

\begin{tabular}{lll} 
Total & 133 & 100,0 \\
\hline
\end{tabular}

Escolaridade

Analfabeto $\quad 5 \quad 4,0$

Ensino fundamental incompleto $50 \quad 40,0$

Ensino fundamental completo $\quad 10 \quad 8,0$

$\begin{array}{lll}\text { Ensino médio incompleto } & 8 & 6,4\end{array}$

Ensino médio completo $30 \quad 24,0$

Ensino superior incompleto $\quad 4 \quad 3,2$

$\begin{array}{lll}\text { Ensino superior completo } & 18 & 14,4\end{array}$

$\begin{array}{lll}\text { Total } & 125 \quad 100,0\end{array}$

$\begin{array}{lll}\text { Omissos } & 8 & 6,0\end{array}$

\section{Comorbidades}

Não possui 30

Hipertensão arterial sistêmica $\quad 34 \quad 30,9$

$\begin{array}{lll}\text { Hipotireoidismo } & 8 & 7,3\end{array}$

$\begin{array}{lll}\text { Psoríase } & 3 & 2,7\end{array}$

$\begin{array}{lll}\text { Diabetes mellitus } & 13 & 11,8\end{array}$

$\begin{array}{lll}\text { Dislipidemia } & 5 & 4,5\end{array}$

$\begin{array}{lll}\text { Outros } & 17 & 15,4\end{array}$

$\begin{array}{lll}\text { Total } & 110 \quad 100,0\end{array}$

$\begin{array}{lll}\text { Omissos } & 23 & 17,2\end{array}$

\section{Estado civil}

\begin{tabular}{lcc} 
Solteiro & 27 & 21,1 \\
Casado & 72 & 56,3 \\
Viúvo & 10 & 7,8 \\
União estável & 6 & 4,7 \\
Divorciado & 13 & 10,2 \\
Total & 128 & 100,0 \\
Omissos & 5 & 3,8 \\
\hline Renda familiar & & \\
1 a 3 salários mínimos & 58 & 77,3 \\
3 a 5 salários mínimos & 13 & 17,3 \\
Mais de 5 salários mínimos & 4 & 5,3 \\
Total & 75 & 100,0 \\
Omissos & 58 & 43,6 \\
\hline
\end{tabular}

Legendas: $\mathrm{N}=$ número de participantes; \%=frequência relativa; *dado expresso em média \pm desvio-padrăo. 
Tabela 2. Características clínicas do estadiamento do câncer $(n=133)$

\begin{tabular}{l|c|c}
\hline Estadiamento T & $\mathbf{N}$ & $\%$ \\
\hline T0 & 2 & 1,9 \\
T1 & 26 & 24,3 \\
T2 & 33 & 30,8 \\
T3 & 22 & 20,6 \\
T4 & 18 & 16,8 \\
TX & 6 & 5,6 \\
Total & 107 & 100,0 \\
Omissos & 26 & 19,5 \\
\hline Estadiamento $\mathbf{N}$ & $\mathbf{N}$ & $\%$ \\
\hline N0 & 32 & 29,9 \\
N1 & 16 & 15,0 \\
N2 & 44 & 41,1 \\
N3 & 8 & 7,5 \\
NX & 7 & 6,5 \\
Total & 107 & 100,0 \\
Omissos & 26 & 19,5 \\
\hline Estadiamento $\mathbf{M}$ & $\mathbf{N}$ & $\%$ \\
\hline 0 & 56 & 60,9 \\
1 & 3 & 3,3 \\
MX & 33 & 35,9 \\
Total & 92 & 100,0 \\
Omissos & 41 & 30,8 \\
\hline
\end{tabular}

Legendas: $\mathrm{N}=$ número de participantes; \%=frequência relativa.

Tabela 3. Características clínicas dos pacientes com câncer de cabeça e pescoço $(n=133)$

\begin{tabular}{lcc}
\hline Tipo de câncer & $\mathbf{N}$ & $\%$ \\
\hline Cavidade oral & 35 & 26,32 \\
Tireoide & 34 & 25,56 \\
Orofaringe & 32 & 24,06 \\
Laringe & 13 & 9,77 \\
Nasofaringe & 7 & 5,26 \\
Hipofaringe & 5 & 3,76 \\
Outros & 7 & 5,25 \\
Total & 133 & 100,0 \\
\hline Metástase & $\mathbf{N}$ & $\%$ \\
\hline Sim & 25 & 18,8 \\
Não & 108 & 81,2 \\
Total & 133 & 100,0 \\
\hline Local da metástase & $\mathbf{N}$ & $\%$ \\
\hline Cervical & 11 & 42,3 \\
Pulmonar & 3 & 11,5 \\
Glândulas salivares & 2 & 7,7 \\
Outros & 10 & 38,5 \\
Total & 26 & 100,0 \\
Omissos & 107 & 80,5 \\
\hline
\end{tabular}

Legendas: $\mathrm{N}=$ número de participantes; \%=frequência relativa.
Tabela 4. Características dos tratamentos clínico e cirúrgico $(n=133)$

\begin{tabular}{lcc}
\hline Realizaram radioterapia & $\mathbf{N}$ & $\%$ \\
\hline Sim & 102 & 76,7 \\
Não & 31 & 23,3 \\
Total & 133 & 100,0 \\
\hline Local da incidência & $\mathbf{N}$ & $\%$ \\
\hline Leito tumoral e drenagem cervical & 9 & 15,8 \\
Região cervical & 7 & 12,3 \\
Cervicofaciais e drenagem cervical & 7 & 12,3 \\
Cervicofaciais & 4 & 7,0 \\
Leito operatório e drenagem & 4 & 7,0 \\
cervical & 3 & 5,3 \\
Lesão primária e drenagens eletivas & 33 & 40,3 \\
Outros & 23 \\
Total & 57 & 100,0 \\
Omissos & 76 & 57,1 \\
\hline Realizaram quimioterapia & $\mathbf{N}$ & $\%$ \\
\hline Sim & 71 & 56,8 \\
Não & $\mathbf{N}$ & $\%$ \\
\hline Total & 111 & 83,5 \\
Omissos & 22 & 16,5 \\
\hline Realizaram radioiodoterapia & $\mathbf{N}$ & $\%$ \\
\hline Sim & 133 & 100,0 \\
\hline Não & 117 & 88,0 \\
Total & 125 & 100,0 \\
\hline Realizaram cirurgia & 8 & 6,0 \\
\hline Sim & 133 & 100,0 \\
\hline Não & 73,2 \\
\hline Total & 73,0 \\
\hline
\end{tabular}

Legendas: $\mathrm{N}=$ número de participantes; \%=frequência relativa.

Diversas são as complicaçóes físicas apresentadas relacionadas aos tratamentos clínico e cirúrgico. Após a radioterapia, observaram-se complexidades relacionadas ao tratamento, com maior incidência da radiodermite $(82,7 \%, n=81)$, seguida pela odinofagia $(69,4 \%, n=68)$ e mucosite $(68,4 \%, n=67)$. Após a quimioterapia, as principais queixas e complicaçôes apresentadas pelos pacientes foram náuseas $(81 \%, \mathrm{n}=34)$ e vômitos $(52,4 \%, n=22)$ (Tabela 3). Após a cirurgia, durante o acompanhamento pós-operatório, foram observadas com maior frequência fibroses no local da incisão cirúrgica $(18,2 \%, \mathrm{n}=10)$ (Tabela 5).

\section{DISCUSSÃO}

Verificou-se, após os resultados do estudo, que o câncer de cabeça e pescoço é ainda muito incidente na 
Tabela 5. Principais complicações apresentadas após os tratamentos clínico e cirúrgico $(n=133)$

\begin{tabular}{|c|c|c|}
\hline $\begin{array}{l}\text { Complicações da } \\
\text { radioterapia }\end{array}$ & $\mathbf{N}$ & $\%$ \\
\hline Radiodermite & 81 & 82,7 \\
\hline Xerostomia & 55 & 56,1 \\
\hline Odinofagia & 68 & 69,4 \\
\hline Disfagia & 56 & 57,1 \\
\hline Disgeusia & 24 & 24,5 \\
\hline Mucosite & 67 & 68,4 \\
\hline $\begin{array}{l}\text { Candidíase } \\
\text { pseudomembranosa }\end{array}$ & 13 & 13,3 \\
\hline Náuseas & 11 & 11,2 \\
\hline Trismo & 8 & 8,2 \\
\hline Vômitos & 6 & 6,1 \\
\hline $\begin{array}{l}\text { Complicações da } \\
\text { quimioterapia }\end{array}$ & $\mathbf{N}$ & $\%$ \\
\hline Náuseas & 34 & 81,0 \\
\hline Vômitos & 22 & 52,4 \\
\hline Mal-estar & 6 & 14,3 \\
\hline Astenia & 9 & 21,4 \\
\hline Alopecia & 4 & 9,5 \\
\hline Epigastralgia & 3 & 7,1 \\
\hline Cefaleia & 4 & 9,5 \\
\hline Alteração no paladar & 3 & 7,1 \\
\hline Neuropatia & 3 & 7,3 \\
\hline $\begin{array}{l}\text { Complicações do } \\
\text { tratamento cirúrgico }\end{array}$ & Frequência & $\%$ \\
\hline Fibrose & 10 & 18,2 \\
\hline Trismo & 4 & 7,3 \\
\hline Lesão do nervo acessório & 2 & 3,6 \\
\hline Seroma & 2 & 3,6 \\
\hline $\begin{array}{l}\text { Paralisia da prega vocal } \\
\text { esquerda }+ \text { fibrose }\end{array}$ & 2 & 3,6 \\
\hline $\begin{array}{l}\text { Paresia do nervo } \\
\text { mandibular à esquerda }\end{array}$ & 2 & 3,6 \\
\hline Edema & 2 & 3,6 \\
\hline Disfonia & 2 & 3,6 \\
\hline Outros & 29 & 52,9 \\
\hline Total & 55 & 100,0 \\
\hline Omissos & 78 & 58,6 \\
\hline
\end{tabular}

Legendas: $\mathrm{N}=$ número de participantes; \%=frequência relativa.

populaçáo, caracterizando a doença como um problema de saúde pública.

No estudo de Casati et al. ${ }^{13}$, tal tipo de câncer já se demonstrava de forma prevalente principalmente em países de menor nível socioeconômico e associado à alta mortalidade ${ }^{13}$, apresentando-se com crescimento progressivo, apesar de a designaçáo dos dados ser subestimada em virtude das falhas no registro de dados ${ }^{13,14}$.

O presente estudo se mostrou de acordo com os dados da literatura brasileira em relação aos aspectos clínicos e epidemiológicos ${ }^{13,14,16}$. Quanto à predominância de neoplasia de câncer de cabeça e pescoço, o gênero mais acometido foi o sexo masculino, como descrito por alguns autores que verificaram também maior incidência dos casos em pacientes do sexo masculino, corroborando os resultados deste estudo ${ }^{13-17}$.

Em relação à idade dos pacientes, observou-se uma média de 57,9 anos, como também verificado por Sousa et al. ${ }^{14}$, cuja média de idade dos pacientes foi de 60,6 anos, com maior predominância também no sexo masculino. No estudo realizado por Rocha et al. ${ }^{16}$, a média de idade também foi semelhante, 59,78 $( \pm 12,49)$ anos. A idade dos pacientes relatada no presente estudo está de acordo com os estudos realizados sobre o câncer de cabeça e pescoço, mostrando que o número de casos ocorre em maior quantidade após a quinta década de vida ${ }^{14,17-21}$. Quanto às comorbidades mais relevantes, no presente estudo, observou-se um elevado número de pacientes hipertensos e diabéticos, como também visto no estudo de Pereira et al. ${ }^{20}$, no qual a maior comorbidade apresentada pelos pacientes foi a hipertensão arterial.

Conforme verificado e relatado nos resultados apresentados, grande parte dos pacientes era fumante e etilista. O alto consumo de tabaco e álcool é apontado como uma das principais causas de aumento do câncer de cabeça e pescoço ${ }^{22}$, sendo os homens mais comumente expostos ao álcool do que as mulheres ${ }^{19}$. Alguns autores relatam que a continuidade do uso do tabaco após o início do tratamento aumenta o risco de recidiva, como também de um segundo tumor primário. Dos pacientes tratados com essa doença, cerca de $20 \%$ a $50 \%$ permanecem com o hábito de fumar mesmo após o fim do tratamento ${ }^{23}$.

Silveira et al. ${ }^{18}$ relataram que, dos 380 pacientes analisados em seus estudos, a maioria abandonou o hábito de fumar nos cinco anos antecedentes à realização de seu estudo, sendo grande parte deles ex-fumante. No estudo de Galbiatti et al..$^{24}$, verificou-se que, além do álcool e tabaco, outros fatores também influenciam no desenvolvimento dessa doença, sendo alguns deles a infecção por HPV; agentes infecciosos; exposição a agentes carcinogênicos e à luz ultravioleta; como também a atividade profissional. Segundo os autores, interromper o hábito de fumar e o consumo de álcool, fugir da exposição a agentes carcinogênicos ambientais, fazer exames para o HPV e controlar o estresse são algumas medidas para prevenir ou tardar o desenvolvimento do câncer ${ }^{24}$.

O presente estudo traz o maior predomínio quanto aos sítios primários de tumores em cavidade oral, como 
também descrito em grande parte da literatura ${ }^{2,13,18,23,25}$. Já outros estudos mostram predomínio em laringe, seguido por cavidade oral, o que difere do achados deste estudo ${ }^{14,16}$, no qual $30,8 \%$ dos pacientes representam a categoria T2 (tumores entre 2 e $4 \mathrm{~cm})^{26}, 41,1 \%$ identificaram um grande número de pacientes com metástases na cadeia linfonodal, e 18,8\% apresentaram metástase, mostrando um grau avançado da doença quando diagnosticada, como observado também na literatura ${ }^{13,18}$.

No estudo realizado por Melo et al. ${ }^{19}$, o estadiamento TNM apresentou maior prevalência em T4 (tumores que invadem estruturas adjacentes ${ }^{26}$ e $38,3 \%$ apresentaram metástases linfonodais. Esse tipo de metástase é comum entre os pacientes com câncer de cabeça e pescoço, ocorrendo com grande incidência. Quando a doença é diagnosticada na fase inicial, sendo menor que $2 \mathrm{~cm}$ de extensão e sem comprometimento linfonodal, o tratamento cirúrgico certamente será suficiente para curar o paciente, mas o diagnóstico feito tardiamente possibilita um aumento da extensáo local, havendo ou não invasão linfonodal, alterando assim o estadiamento, necessitando de tratamento complementar por intermédio da radioterapia, quimioterapia ou ambas ${ }^{27}$.

Quanto aos tratamentos clínico e cirúrgico, neste estudo, grande parte dos pacientes realizou radioterapia em conjunto com o tratamento cirúrgico, conforme descrito na literatura, que traz a associação de ambos os tratamentos na maioria dos casos ${ }^{25}$. Casati et al. ${ }^{13}$ corroboram este estudo, citando que o tratamento do câncer de cabeça e pescoço é complexo e em lesóes mais avançadas é principalmente cirúrgico e normalmente associado à radioterapia, à quimioterapia ou ambas. Alvarenga et al..$^{25}$ citam em seus achados que as indicaçóes para radioterapia ou cirurgia se direcionam para os tumores classificados como T1 e T2 e a maioria dos tumores $\mathrm{T} 3 \mathrm{e}$ T4 necessita de um tratamento multimodal, no qual normalmente se realiza cirurgia seguida pela radioterapia adjuvante.

Quanto ao tratamento, o uso de cirurgia, radioterapia, quimioterapia ou tratamento associado dependerá da ressecabilidade e localização do tumor; a principal opção de tratamento para doença primária é a cirurgia. $\mathrm{O}$ tratamento da quimioterapia e da radioterapia é uma abordagem viável e proporciona maior sobrevida aos pacientes, no entanto, aplicadas em altas doses, estas podem levar a efeitos colaterais, interferindo na qualidade de vida e afetando funçóes como a mastigação, a deglutição e até mesmo a fala ${ }^{9,24}$. Assim como verificado neste estudo, o procedimento cirúrgico foi o tratamento mais realizado, sendo normalmente associado à radioterapia, à quimioterapia ou a ambas.

Diversas são as complicaçôes apresentadas pelos tratamentos clínico e cirúrgico, observadas no presente estudo, no qual a radioterapia gerou em grande parte dos pacientes alteraçôes importantes. Nos estudos de Campos e Leite ${ }^{28}$ e Rodrigues ${ }^{29}$, foram observadas complicações orais comuns, com início geralmente durante ou após radioterapia, sendo algumas delas a mucosite, xerostomia, cáries, disgeusia, infecçôes secundárias, osteorradionecrose e trismo. Algumas das complicaçóes apresentadas pelos autores vão ao encontro dos resultados, entretanto, no presente estudo aparecem com menor incidência, não sendo as mais relevantes. Tais complicaçóes variam de acordo com a área irradiada, dose total, quantidade de radiação administrada e das condiçóes clínicas do paciente $^{30}$.

No estudo realizado por Sawada et al..$^{31}$, os resultados encontrados pelos autores são semelhantes aos observados nesta pesquisa, e trazem relatos dos pacientes que mostram um elevado número de queixas geradas pós-tratamento radioterápico, entre elas, coceiras na pele, boca seca, dificuldade ou dor na deglutição e alteraçôes no paladar.

As complicaçóes geradas pelos procedimentos cirúrgicos mais prevalentes encontradas no presente estudo foram fibroses. A literatura mostra que as complicaçôes dependerão da técnica utilizada e da extensão da lesão, podendo ocorrer fibroses, parestesias em ombros e pescoço e deformidades que irão afetar a função e a estética facial, podendo haver reconstruçôes por meio da colocação de enxertos ${ }^{32,33}$.

Há deficits que podem ser encontrados em pesquisas epidemiológicas e em especial nessa população. Nesta pesquisa, as informaçóes produzidas a partir do registro hospitalar dos indivíduos do Sul do país, ocasionalmente, apresentaram fragilidades em razão da não completude dos dados, faltando em alguns prontuários hábitos de vida, fatores de risco para o desenvolvimento da doença, comorbidades associadas, diagnóstico anterior de câncer sem tratamento e ausência de história familiar de câncer. Com isso, obtiveram-se informaçóes diminuídas sobre os pacientes e altos números de dados omissos.

\section{CONCLUSÃO}

O perfil clínico-epidemiológico dos pacientes com câncer de cabeça e pescoço está de acordo com os dados descritos na literatura, sendo uma doença composta predominantemente por pacientes do sexo masculino, a partir da quinta década de vida, com baixa escolaridade e renda familiar, sendo majoritariamente tabagistas e etilistas com mais de 30 anos de exposição e tendo como localização primária do tumor mais prevalente a região da cavidade oral.

É de extrema importância conhecer e estudar o perfil epidemiológico dos pacientes com câncer de cabeça e 
pescoço para uma maior análise quanto à deteç̧ão e ao comportamento da doença, conhecimento das possíveis complicaçóes dos tratamentos clínico e/ou cirúrgico, e possibilitar, dessa maneira, uma maior qualidade e o direcionamento das açôes para gerar aprimoramentos nos tratamentos realizados.

\section{CONTRIBUIÇÕES}

Todos os autores contribuíram substancialmente na concepção e no planejamento do estudo, na obtenção, análise e/ ou interpretação dos dados, assim como na redação e/ou revisão crítica e aprovaram a versão final a ser publicada.

\section{DECLARAÇÃO DE CONFLITO DE INTERESSES}

Nada a declarar.

\section{FONTES DE FINANCIAMENTO}

Não há.

\section{REFERÊNCIAS}

1. Döbrossy L. Epidemiology of head and neck cancer: magnitude of the problem. Cancer Metastasis Rev. 2005;24(1):9-17. doi: https://doi.org/10.1007/s10555005-5044-4

2. Bragante KC, Nascimento DM, Motta NW. Avaliação dos efeitos agudos da radioterapia sobre os movimentos mandibulares de pacientes com câncer de cabeça e pescoço. Rev Bras Fisioter. 2011;16(2):141-147. doi: https://doi.org/10.1590/S1413-35552012005000021

3. Marur S, Forastiere AA. Head and neck cancer: changing epidemiology, diagnosis, and treatment. Mayo Clin Proc. 2008;83(4):489-501. doi: https://doi. org/10.4065/83.4.489

4. Wang $\mathrm{M}$, Chu $\mathrm{H}$, Zhang Z, et al. Molecular epidemiology of DNA repair gene polymorphisms and head and neck cancer. J Biomed Res. 2013;27(3):179-92. doi: https:// doi.org/10.7555/JBR.27.20130034

5. Hashibe M, Boffetta P, Zaridze D, et al. Evidence for an important role of alcohol-and aldehyde-metabolizing genes in cancers of the upper aerodigestive tract. Cancer Epidemiol Biomarkers Prev. 2006;15(4): 696-703. doi: https://doi.org/10.1158/1055-9965.EPI-05-0710

6. Gonçalves RP, Bonani FA, Santos RV, et al. Fatores de risco para carcinoma bem diferenciado de tireoide: análise crítica de 463 casos. Rev Bras Cir Cabeça Pescoço. 2012;41(1):23-26.

7. Della Justina LB, Dias M. Head and neck lymphedema: what is the physical therapy approach? A literature review. Fisioter Mov. 2016;29(2):411-9. doi: https://doi. org/10.1590/0103-5150.029.002.AO20

8. Leite MAC, Nogueira DA, Terra FS. Avaliação da autoestima em pacientes oncológicos submetidos a tratamento quimioterápico. Rev Latino-Am Enfermagem. 2015;23(6):1082-9. doi: https://doi.org/10.1590/01041169.0575 .2652

9. Hunter KU, Jolly S. Clinical review of physical activity and functional considerations in head and neck cancer patients. Support Care Cancer. 2013;21:1475-9. doi: https://doi.org/10.1007/s00520-013-1736-4

10. Mozzini CB, Schuster RC, Mozzini AR. O esvaziamento cervical e o papel da fisioterapia na sua reabilitação. Rev Bras Cancerol. 2007;53(1):55-61.

11. Iyomasa RM, Tagliarini JV, Rodrigues SA, et al. Laryngeal and vocal alterations after thyroidectomy. Braz J Otorhinolaryngol. 2019;85(1):3-10. doi: https://doi. org/10.1016/j.bjorl.2017.08.015

12. Instituto Nacional de Câncer José Alencar Gomes da Silva [Internet]. Rio de Janeiro: INCA; c1996-2020. Estadiamento. [acesso 2019 jun. 27]; [cerca de 2 telas]. Disponível em: wwwl.inca.gov.br/impressao. asp?op $=$ cv\&id $=54$

13. Casati MFM, Vasconcelos JA, Vergnhanini GS, et al. Epidemiologia do câncer de cabeça e pescoço no Brasil: estudo transversal de base populacional. Rev Bras Cir Cabeça Pescoço. 2012;41(4):186-91.

14. Sousa AR, Koury GVH, Badaranne EBL, et al. Perfil clínico-epidemiológico de pacientes com câncer de cabeça e pescoço em hospital de referência. Rev Soc Bras Clin Med. 2016;14(3):129-32.

15. Felippu AWD, Freire EC, Silva RA, et al. Impact of delay in the diagnosis and treatment of head and neck cancer. Braz J Otorhinolaryngol. 2016;82(2):140-3. doi: https:// doi.org/10.1016/j.bjorl.2015.10.009

16. Rocha BQC, Eneas L, Oliveira RG, et al. Características epidemiológicas de pacientes portadores de neoplasias de cabeça e pescoço submetidos à radioterapia em Juiz de Fora - MG. HU Rev. 2017;43(1):71-75. doi: https:// doi.org/10.34019/1982-8047.2017.v43.2644

17. Verás ID, Santos AF, Ferreira SMS, et al. Alteraçóes orais e ingestão alimentar em pacientes com câncer de cabeça e pescoço em tratamento antineoplásico. Diversitas J. 2019;4(2):566-79. doi: https://doi.org/10.17648/ diversitas-journal-v4i2.760

18. Silveira A, Gonçalves J, Sequeira T, et al. Oncologia de cabeça e pescoço: enquadramento epidemiológico e clínico na avaliação da qualidade de vida relacionada com a saúde. Rev Bras Epidemiol. 2012;15(1):38-48. doi: https://doi.org/10.1590/S1415-790X2012000100004

19. Melo LC, Silva MC, Bernardo JMP, et al. Perfil epidemiológico de casos incidentes de câncer de boca e faringe. Rev Gaúch Odontol. 2010;58(3):351-5. 
20. Pereira IF, Noronha VRAS, Naves MD, et al. Neoplasias malignas em regiáo de cabeça e pescoço: perfil dos pacientes atendidos na UFMG. Rev Cubana Estomatol. 2016;53(4).

21. Miranda MEP. Prevalência do câncer de cabeça e pescoço no Hospital de Especialidades "Eugenio Espejo" período 2002 - 2015, Quito - Equador [tese na Internet]. São Paulo, SP: Universidade de São Paulo, Faculdade de Odontologia; 2018. 79f. doi: https://doi. org/10.11606/T.23.2019.tde-15032019-145601

22. Machiels JP, Lambrecht M, Hanin FX, et al. Advances in the management of squamous cell carcinoma of the head and neck. F1000Prime Rep. 2014;6:44. doi: https://doi. org/10.12703/P6-44

23. Pinto FR, Matos LL, Segundo WG, et al. Manutenção do tabagismo e etilismo em pacientes tratados por câncer de cabeça e pescoço: influência do tipo de tratamento oncológico empregado. Rev Assoc Med Bras. 2011;57(2):171-6. doi: https://doi.org/10.1590/S010442302011000200014

24. Galbiatti ALS, Padovani-Junior JA, Maníglia JV, et al. Head and neck cancer: causes, prevention and treatment. Braz J Otorhinolaryngol. 2013;79(2):239-47. doi: https://doi.org/10.5935/1808-8694.20130041

25. Alvarenga LM, Ruiz MT, Pavarino-Bertelli EC, et al. Avaliação epidemiológica de pacientes com câncer de cabeça e pescoço em um hospital universitário do Noroeste do Estado de Sáo Paulo. Rev Bras Otorrinolaringol. 2008;74(1):68-73. doi: https://doi. org/10.1590/S0034-72992008000100011

26. Ministério da Saúde (BR). TNM: Classificação de Tumores Malignos. 6. ed. Rio de Janeiro: INCA; 2004.

27. Santos VC, Assis AM, Silva LE, et al. Câncer de boca: análise do tempo decorrido da detecçáo ao início do tratamento em centro de Oncologia de Maceió. Rev Bras Odontol. 2012;69(2):159-64. doi: http://dx.doi. org/10.18363/rbo.v69n2.p.159

28. Campos RJDS, Leite ICG. Qualidade de vida e voz pós-radioterapia: repercussóes para a fonoaudiologia. Rev CEFAC. 2010;12(4):671-7. doi: https://doi. org/10.1590/S1516-18462010005000038

29. Rodrigues AGM. Complicaçóes bucais da radioterapia em cabeça e pescoço [monografia]. Cuiabá, MT: Universidade de Cuiabá, Curso de Odontologia; 2018. 21 f.

30. Oliveira VDP, Aires DMP. Complicaçôes bucais da radioterapia no tratamento do câncer de cabeça e pescoço. REFACER. 2018;7(1):69-86.

31. Sawada NO, Dias AM, Zago MMF. O efeito da radioterapia sobre a qualidade de vida dos pacientes com câncer de cabeça e pescoço. Rev Bras Cancerol. 2006;52(4):323-9.

32. Silvestre-Donat FJ, Puente Sandoval A. Efectos adversos del tratamiento del cáncer oral. Av Odontoestomatol. 2008;24(1):111-121.
33. Qu N, Zhang TT, Wen SS, et al. The application of selective neck dissection while preserving the cutaneous branches of cervical plexus in the surgical treatment of differentiated thyroid cancer-experiences from thousands of cases. Ann Transl Med. 2019;7(7):151. doi: https:// doi.org/10.21037/atm.2019.03.03

Recebido em 9/9/2019 Aprovado em 3/2/2020 